\title{
Beam dynamics in a spectrometer for the polarized positron production experiment ${ }^{*}$
}

\author{
Yuri .K. Batygin \\ SLAC, Stanford University, Stanford, CA 94309
}

\begin{abstract}
The proposed experiment E-166 at SLAC is designed to demonstrate the possibility of producing longitudinally polarized positrons from circularly polarized photons to be used in future Linear Collider. The experimental set-up utilizes a low emittance $50 \mathrm{GeV}$ electron beam passing through a helical undulator in the Final Focus Test Beam line of the SLAC accelerator. Circularly polarized photons generated by the electron beam in the undulator hit a target and produce electron-positron pairs. The purpose of the post-target spectrometer is to select the positron beam and to deliver it to a polarimeter whilst keeping the positron beam polarization as high as possible. This paper analyzes positron transmission and polarization in the E-166 spectrometer experiment. The positron transmission has a maximum value of $7 \%$ for a positron beam energy of $5.5 \mathrm{MeV}$, while positron polarization is approximately $60 \%$.
\end{abstract}

To be published in Review of Scientific Instruments

*Work supported by Department of Energy contract DE-AC02-76SF00515 


\section{INTRODUCTION}

Operation of the future International Linear Collider (ILC) with polarized electron and positron beams will significantly improve the precise measurements in new physics and this is equivalent to an increase of the effective luminosity of the collider $[1,2]$. While polarized electron beams have been steadily operated at SLAC [3] for some time, production of polarized positrons is in the research stage [4]. The traditional method of positron beam polarization used in HERA collider in DESY [5] via Sokolov-Ternov mechanism is not suitable for a linear collider because of the long time required for the beam polarization in a synchrotron. The original method of polarized positron beam production for linear collider was proposed by V.E.Balakin and A.A.Michailichenko in 1979 [6]. In this scheme, a high-energy electron beam passes through a helical undulator and produces circularly polarized photons, which then impinge on a target and produce longitudinally polarized positrons.

The proposed positron production experiment E-166 [7] at SLAC is aimed to demonstrate the possibility of polarized positrons production for a linear collider (see Fig. 1). It uses a strongly collimated $50 \mathrm{GeV}$ electron beam in the SLAC Final Focus Test Beam (FFTB) line to generate circularly polarized photons in a $1 \mathrm{~m}$ long helical undulator. Photons, after interaction with target, create polarized positrons via pair production. The important part of the experiment is the spectrometer, which is used to separate the positron beam after the target from electron and photon beams and to deliver positrons to a reconversion target whilst keeping beam polarization as high as possible. This paper describes positron beam dynamics in the spectrometer both analytically and numerically and determines optimal condition for beam transport. 


\section{SPECTROMETER WITH DOUBLE $90^{\circ}$ MAGNETS}

Non-dispersive spectrometers are widely used in beam optics [8]. Usually, they include two or three bending magnets with additional quadrupoles between them. In the proposed experiment E-166, the spectrometer is required to shift the beam from an original accelerator axis at the distance of $45 \mathrm{~cm}$ to separate the positron beam from a photon beam and from an electron beam coming out from the target. The simple, and cost-effective solution, is to utilize two $90^{\circ}$ magnets providing point-to-point transformation of the beam in the horizontal direction. The parameters of the system are presented in Table 1. An additional feature of the double $90^{\circ}$ magnets design is absence of the depolarization of positrons in the bending magnets, because spin rotation in the first magnet is compensated by the second magnet.

The initial distribution of positrons produced by circularly polarized photons has been calculated by J.C.Sheppard using the program EGS4, modified for polarized positrons [9, 10]. An illustration of the positron distribution after impinging upon the target is presented in Fig. 2. Each FFTB electron bunch with $1 \times 10^{10}$ electrons generates $4 \times 10^{9}$ photons, which, after interaction with $0.5 \mathrm{RL}$ Ti target produce $\mathrm{N}_{\mathrm{e}+}=2 \times 10^{7}$ positrons [7]. The distribution of positrons is characterized by a large emittance of the positron beam and a correspondingly large energy spread. We define the polarization of particle as:

$$
\mathrm{P}=\frac{\mathrm{P}_{+}-\mathrm{P}_{-}}{\mathrm{P}_{+}+\mathrm{P}_{-}}
$$

where $\mathrm{P}_{+}$and $\mathrm{P}_{-}$are number of positrons with spin along and opposite to the direction of particle momentum, respectively.

The initial distribution is correlated in energy, polarization and transverse momentum spread of positrons (see Figs. 2, 3). The low energy positrons are less polarized and more transversely divergent, while high-energy positrons are strongly polarized and less divergent. The energy spectrum peaks near the low-energy end of the distribution. As a consequence of a specific correlation between positrons 
polarization and energy, there is an inevitable compromise between number of accepted positrons and beam polarization.

Due to a long tail in the energy spectrum of the initial positron distribution, it is desirable to design a beamline which accepts positrons with large momentum spread. Multiplication of transport matrixes for the proposed bend-drift-inverse bend structure gives in horizontal plane:

$$
\begin{aligned}
& \left|\begin{array}{ccc}
0 & \mathrm{R} & \mathrm{R} \\
-\frac{1}{\mathrm{R}} & 0 & 1 \\
0 & 0 & 1
\end{array}\right|\left|\begin{array}{ccc}
1 & \mathrm{~L} & 0 \\
0 & 1 & 0 \\
0 & 0 & 1
\end{array}\right|\left|\begin{array}{ccc}
0 & \mathrm{R} & -\mathrm{R} \\
-\frac{1}{\mathrm{R}} & 0 & -1 \\
0 & 0 & 1
\end{array}\right|, \quad \text { or } \\
& \left|\begin{array}{c}
\mathrm{x} \\
\mathrm{x}^{\prime} \\
\mathrm{dp} / \mathrm{p}
\end{array}\right|=\left|\begin{array}{ccc}
-1 & 0 & 0 \\
\frac{\mathrm{L}}{\mathrm{R}^{2}} & -1 & -\left(2+\frac{\mathrm{L}}{\mathrm{R}}\right) \\
0 & 0 & 1
\end{array}\right| \mid \begin{array}{c}
\mathrm{x} \\
\mathrm{x}^{\prime} \\
\mathrm{dp} /\left.\mathrm{p}\right|_{0}
\end{array}
\end{aligned}
$$

From the matrix, Eq (2.3), it is clear that the final position of particles to a linear approximation is momentum-independent. The maximum horizontal displacement of the particle is achieved after the first bend and drift, and is given by:

$$
x=-\frac{L}{R} x_{O}+R x_{O}^{\prime}+(R+L) \frac{\Delta p}{p_{o}}
$$

The maximum deviation from the axis is equal to the radial aperture, $\mathrm{a}_{\mathrm{x}}=5 \mathrm{~cm}$. Referring to Eq. (2.4) the horizontal acceptance of the channel, $\varepsilon_{\mathrm{x}}$, is defined through initial maximum deviation, $\mathrm{x}_{0 \text {, max }}$, and maximum divergence, $\mathrm{x}_{\mathrm{O}}^{\prime}$, $\max$, as:

$$
\begin{gathered}
\mathrm{x}_{\mathrm{O}, \max }=\mathrm{a}_{\mathrm{x}} \frac{\mathrm{R}}{\mathrm{L}}=3.125 \mathrm{~cm}, \\
\mathrm{x}_{\mathrm{O}, \max }^{\prime}=\frac{\mathrm{a}_{\mathrm{x}}}{\mathrm{R}}=0.4, \\
\varepsilon_{\mathrm{x}}=\pi \mathrm{x}_{\mathrm{O}, \max } \mathrm{x}_{\mathrm{O}, \max }^{\prime}=1.25 \pi \mathrm{cm} \mathrm{rad} .
\end{gathered}
$$


The maximum momentum spread of transmitted positrons is defined from Eq. (2.4) as:

$$
\left(\frac{\Delta \mathrm{p}}{\mathrm{p}_{\mathrm{o}}}\right)_{\max }=\frac{\mathrm{a}_{\mathrm{x}}}{\mathrm{R}+\mathrm{L}}=0.15
$$

In the vertical direction, particle motion is unaffected apart from the influence of edge defocusing at the entrance and exit of the bending magnets. The maximum vertical slope of particle trajectory is $\mathrm{y}_{\mathrm{o}, \max }^{\prime}=0.027$, and the maximum initial vertical displacement of positrons is $\mathrm{y}_{\mathrm{o}, \max }=0.9 \mathrm{~cm}$. Therefore, the vertical acceptance is

$$
\varepsilon_{\mathrm{y}}=\pi \mathrm{y}_{\mathrm{o}, \max } \mathrm{y}_{\mathrm{o}, \max }^{\prime}=0.024 \pi \mathrm{cm} \mathrm{rad}
$$

An additional feature of the double $90^{\circ}$ magnets design is absence of the depolarization of positrons. The spin precession of positrons in a $\theta=90^{\circ}$ bending magnet for max beam energy of $\gamma_{\max }=20$ is:

$$
\varphi_{\max }=\theta \gamma_{\max } \mathrm{G}=2^{\circ},
$$

where $G=0.001159652$ is the anomalous magnetic moment of positron. However, spin rotation in the first bending magnet is compensated by the second magnet.

Substitution of the constraints, given in Eqs. (2.5) - (2.9), into the initial distribution, enables the maximum value of positron transmission, $\mathrm{N} / \mathrm{N}_{\mathrm{o}}=10^{-2}$ to be obtained, where $\mathrm{N}_{\mathrm{o}}$ is the number of positrons at the target and $\mathrm{N}$ is the number of transmitted positrons. The transmission efficiency in the spectrometer is improved by inserting a solenoid between the positron production target and the bending magnets. The focusing properties of the solenoid lens are characterized by the focal length of the lens, $f$, defined as:

$$
\frac{1}{\mathrm{f}}=\left(\frac{\mathrm{e}}{2 \mathrm{p}_{\mathrm{z}}}\right)^{2} \int_{-\mathrm{d}}^{\mathrm{d}} \mathrm{B}_{\mathrm{z}}^{2} \mathrm{dz},
$$

where $\mathrm{B}_{\mathrm{z}}$ is the longitudinal on-axis component of the magnetic field of the solenoid. The positron momentum is given in terms of the bending field B as: 


$$
\mathrm{p}_{\mathrm{z}}=\mathrm{eBR}
$$

and there is a linear relationship between the values of $B$ and $B_{z}$ to provide optimal focusing of particles along the beamline. An increase of the bending field for transmission of positrons with larger energy requires a proportional increase of the solenoid field.

\section{POLARIZED POSITRON BEAM DYNAMICS}

The proposed spectrometer is characterized by large values of $\mathrm{x} / \mathrm{R} \sim 0.4$ and momentum deviation $\delta=\Delta \mathrm{p} / \mathrm{p} \sim 0.15$. Let us consider a single particle trajectory in a bending magnet with parallel poles. The particle position inside magnet with second order aberration terms is given by [11]

$$
\begin{aligned}
\mathrm{x} & =\mathrm{x}_{\mathrm{o}} \cos \theta+R \sin \theta \mathrm{x}_{\mathrm{o}}^{\prime}+\mathrm{R}(1-\cos \theta) \delta \\
& +\mathrm{T}_{111} \mathrm{x}_{\mathrm{o}}^{2}+\mathrm{T}_{112} \mathrm{x}_{\mathrm{o}} \mathrm{x}_{\mathrm{o}}^{\prime}+\mathrm{T}_{116} \mathrm{x}_{\mathrm{o}} \delta+\mathrm{T}_{122} \mathrm{x}_{\mathrm{o}}^{\prime}{ }^{2}+\mathrm{T}_{126} \mathrm{x}_{\mathrm{o}}^{\prime} \delta+\mathrm{T}_{166} \delta^{2}+\mathrm{T}_{133 \mathrm{y}_{\mathrm{o}}}+\mathrm{T}_{134 \mathrm{y}_{\mathrm{o}} \mathrm{y}_{\mathrm{o}}^{\prime}+\mathrm{T}_{144 \mathrm{y}_{\mathrm{o}}^{\prime}}}{ }^{2}
\end{aligned}
$$

where $T_{i j k}$ are second order aberration coefficients. For magnet with bending angle $\theta=90^{\circ}$, Eq. (3.1) is:

$$
\mathrm{x}=\mathrm{R} \mathrm{x}_{\mathrm{O}}^{\prime}+\mathrm{R} \delta-\frac{\mathrm{x}_{\mathrm{O}}^{2}}{2 \mathrm{R}}+\frac{\mathrm{x}_{\mathrm{O}} \delta}{3}+\mathrm{R} \delta \mathrm{x}_{\mathrm{o}}^{\prime}-\frac{\mathrm{R}}{2} \delta^{2}-\frac{\mathrm{R}}{2} \mathrm{y}_{\mathrm{o}}^{\prime 2}
$$

The positron beam after impinging upon the target contains particles with, $\mathrm{xo}^{\prime} \approx 1, \mathrm{yo}^{\prime} \approx 1$ (see Fig. 2). According to Eq. (3.2), the contribution of higher order terms, $\mathrm{R} \delta \mathrm{x}_{\mathrm{O}}^{\prime}, 0.5 \mathrm{Ry}_{\mathrm{o}}^{\prime}{ }^{2}$, in particle dynamics might be significant because they are of the same order as linear terms, $\mathrm{R} \delta, \mathrm{Rx} \mathrm{x}_{\mathrm{O}}^{\prime}$. The validity of the linear model based on linear matrix multiplication is required to be verified by numerical simulations.

The particle-in-cell code BEAMPATH [12] is used for all the numerical calculations presented herein. Particle trajectories are calculated in a curved system of coordinates which naturally includes 
higher order terms in particle dynamics. Particle tracking is accompanied with integration of the Thomas-BMT equation, describing the precession of the spin vector $\overrightarrow{\mathrm{S}}$ [13]:

$$
\frac{\mathrm{d} \overrightarrow{\mathrm{S}}}{\mathrm{dt}}=\frac{\mathrm{e} \overrightarrow{\mathrm{S}}}{\mathrm{m} \gamma} \times\left[(1+\mathrm{G} \gamma) \overrightarrow{\mathrm{B}}_{\perp}+(1+\mathrm{G}) \overrightarrow{\mathrm{B}}_{\mathrm{II}}+\left(\mathrm{G} \gamma+\frac{\gamma}{1+\gamma}\right) \frac{\overrightarrow{\mathrm{E}} \times \vec{\beta}}{\mathrm{c}}\right]
$$

where $\vec{E}$ is the electrical field, and $\vec{B}_{\perp}$ and $\vec{B}_{I I}$ are components of the magnetic field perpendicular and parallel to particle velocity. Initially, the spin vector of each positron is pointed along momentum vector. During beam transport, the spin vector precesses. We define the longitudinal polarization as an average of the product of the longitudinal component $\mathrm{S}_{\mathrm{Z}}$ and the value of polarization, $\mathrm{P}$, summed over all positrons:

$$
<\mathrm{P}_{\mathrm{z}}>=\frac{1}{\mathrm{~N}} \sum_{i=1}^{\mathrm{N}} \mathrm{S}_{\mathrm{z}}^{(\mathrm{i})} \mathrm{P}^{(\mathrm{i})}
$$

The initial value of longitudinal polarization of the beam is $\left\langle\mathrm{P}_{\mathrm{Z}}\right\rangle=0.41$. The low value of polarization is explained by the presence of a large number of low-energy, poorly polarized positrons in the beam (see Fig. 2). After removing these low-energy positrons in the spectrometer, the final beam polarization can reach 0.6 to 0.8 .

To estimate the effect of space charge on beam dynamics, consider the equation for a round beam envelope, $R_{x}$, in free space [14]:

$$
\frac{d^{2} R_{x}}{d z^{2}}-\frac{\ni^{2}}{R_{x}^{3}}-\frac{2 I}{R_{x}(\beta \gamma)^{3} I_{c}}=0
$$

where $\ni$ is the natural beam emittance, $I=\mathrm{eN}_{\mathrm{e}+} \mathrm{f}_{\mathrm{RF}}$ is the beam current, $\mathrm{f}_{\mathrm{RF}}$ is the linac operational frequency, and $I_{c}=4 \pi \varepsilon_{0} \mathrm{mc}^{3} / \mathrm{e}=17 \mathrm{kA}$ is the characteristic current. Here, beam defocusing is defined by two terms in Eq. (3.5): one is proportional to square of beam emittance and the other is proportional 
to the beam current. The ratio of these terms gives an estimation of which phenomena dominates the beam dynamics:

$$
\mathrm{b}=\frac{2}{(\beta \gamma)^{3}} \frac{\mathrm{I}}{\mathrm{I}_{\mathrm{c}}}\left(\frac{\mathrm{R}_{\mathrm{x}}}{\ni}\right)^{2}
$$

Utilizing the numerical values for the proposed experiment, namely, $f_{\mathrm{RF}}=2.856 \times 10^{9} \mathrm{~Hz}, \mathrm{I}=9,1 \times 10^{-3} \mathrm{~A}$, $\ni=0.002 \pi \mathrm{m} . \mathrm{rad}, \beta \gamma=7, \mathrm{R}_{\mathrm{x}}=0.3 \times 10^{-2} \mathrm{~m}$, in Eq. (3.6) gives: $\mathrm{b}=7 \times 10^{-9}$. This indicates that space charge forces are negligible with respect to defocusing due to beam emittance.

Particle trajectories in the proposed system are illustrated in Fig. 4. Curves of the longitudinal positron beam polarization, $\left\langle\mathrm{P}_{\mathrm{z}}\right\rangle$, and average value of longitudinal spin component, $\mathrm{S}_{\mathrm{z}}$, are presented in Fig. 5. It is interesting to note that both of these parameters are increasing along the structure and this is due to a filtration of low-energy, poorly polarized positrons in the spectrometer.

Positron transmission and polarization after transport system as functions of beam energy are illustrated in Fig. 6. These curves are obtained by varying the magnetic field in both magnets and this provides transmission of positrons, with average momentum defined by Eq. (2.12). Positrons were transmitted numerically through a solenoidal field and then either a numerical integration of trajectories was applied or an analytical model based on Eqs. (2.5) - (2.9) was used. A comparison between analytical and numerical approaches indicates that both methods give similar results. The appearance of a maximum in the positron transmission versus beam energy is explained by the correlation between positron energy and divergence (see Fig. 3). In the low-energy part of spectrum, the number of positrons is large, however only a small fraction of positrons is within the transverse acceptance of the spectrometer. As a result, the number of transmitted low-energy positrons is small. As the energy increases, the positron beam becomes less divergent and transmission is better. At the same time, the number of positrons drops with energy and at the high-energy part of spectrum, the transmission is also small. An optimal value of the magnetic field of the spectrometer is 0.15 Tesla, 
which provides the best transmission and a relatively high value of positron polarization (for positrons with average energy of $5.5 \mathrm{MeV}$ ).

Due to absence of vertical focusing, most of particle losses are observed in vertical direction. Utilizing non-parallel poles in the bending magnets increases the vertical focusing and decreases the radial focusing. A magnetic field with non-parallel poles is characterized by the field index

$$
\mathrm{n}=-\left[\frac{\mathrm{R}}{\mathrm{B}_{\mathrm{y}}} \frac{\partial \mathrm{B}_{\mathrm{y}}}{\partial \mathrm{x}}\right]_{\mathrm{x}=0, \mathrm{y}=0},
$$

where $\mathrm{B}_{\mathrm{y}}$ is the vertical field component at the axis. Fig. 7 illustrates transmission efficiency as a function of field index. Here the positron transmission efficiency has a maximum of $7 \%$ at the value of $n=$ $0.3 \ldots 0.4$. The pole surface is described by the equation [15]:

$$
y=\frac{R a_{y}}{n} \frac{1}{\left(\frac{R}{n}-x\right)} .
$$

Eq. (3.8) describes a hyperbolic surface. This can be well approximated by straight poles

$$
\frac{d y}{d x}=-\frac{R a_{y}}{n} \frac{1}{\left(\frac{R}{n}-x\right)^{2}}=n \frac{a_{y}}{R}=0.096,
$$

which eventually provide the best transmission of positrons in spectrometer.

\section{CONCLUSIONS}

A spectrometer for polarized positrons production experiment is proposed. It consists of double $90^{\circ}$ bending magnets and a solenoid. The design presented herein was based on a compromise which required a simple solution to enable the transmission of a positron beam with large emittance whilst maintaining the highest possible positron polarization. Positron transmission as a function of positron beam energy has a maximum while beam polarization increases with energy of transmitted positrons. 
The optimal setting of the spectrometer maximizes positron transmission at the value of $7 \%$ with a beam polarization of $60 \%$.

\section{ACKNOWLEDGEMENTS}

The author is indebted to Dr R.M. Jones for carefully reading the manuscript and for some editorial comments.

\section{REFERENCES}

1. T.Omori “A polarized positron beam for linear colliders”, KEK Preprint 98-237, 1999.

2. G.Moortgat-Pick and H.Steiner, "Physics Opportunities with Polarized e- and e+ Beams at TESLA", LC-TH-2000-055, DESY 00-178.

3. H.Tang, et al, "The SLAC Polarized Electron Source", International Workshop on Polarized Beams and Polarized Gas Targets, Cologne, Germany, June 6-9 , 1995, SLAC-PUB-6918.

4. V.Strakhonenko, et al., NIM-A 547, p.p, 320-333, 2005.

5. D.Barber, E.Gianfelice-Wendt, Physics World, p.p. 24-25, July 2003.

6. V.E.Balakin, A.A.Michailichenko, "The Conversion system for obtaining high polarized electrons and positrons", INP 79-85, Novosibirsk, 1979.

7 ."Undulator-based production of polarized positrons. A proposal for the 50-GeV beam in the FFTB”, SLAC-NT-04-018, 2004

.8. K.G.Steffen, “High Energy Beam Optics”, Interscience Publishing, 1965.

9. W.Nelson, H.Hirayama and D.Rogers, "The EGS4 Code System", SLAC-Report-265,1985.

10. K.Flottmann, Ph.D. Thesis, DESY-93-161A, 1993.

11. K.L.Brown, SLAC-75, 1982.

12. Y.K.Batygin, NIM-A 539, p. p. 455 - 489, 2005.

13. V.Bargmann, L.Michel, V.L.Telegdi, Phys. Rev. Lett. 2, 435, 1959. 
14. I.M.Kapchinsky, “Theory of Resonance Linear Accelerators”, Hawood, New York, 1985.

15. "Handbook of Accelerator Physics and Engineering", Editors: A.Chao and M.Tigner, World Scientific, 1999, p.439. 
Table 1. Parameters of the structure

Bending radius, $\mathrm{R}$

Bending angle

Gap, g

Fringe field coefficients: $\mathrm{K}_{1}$

$$
\mathrm{K}_{2}
$$

Vertical aperture, $2 \mathrm{a}_{\mathrm{y}}$

Horizontal aperture, $2 \mathrm{a}_{\mathrm{x}}$

Aperture at the exit of transport system

Drift between bending magnets, $\mathrm{L}$
$12.5 \mathrm{~cm}$

$90^{\circ}$

$5 \mathrm{~cm}$

0.7

4.4

$5 \mathrm{~cm}$

$10 \mathrm{~cm}$

$5 \times 5 \mathrm{~cm}^{2}$

$40 \mathrm{~cm}$ 


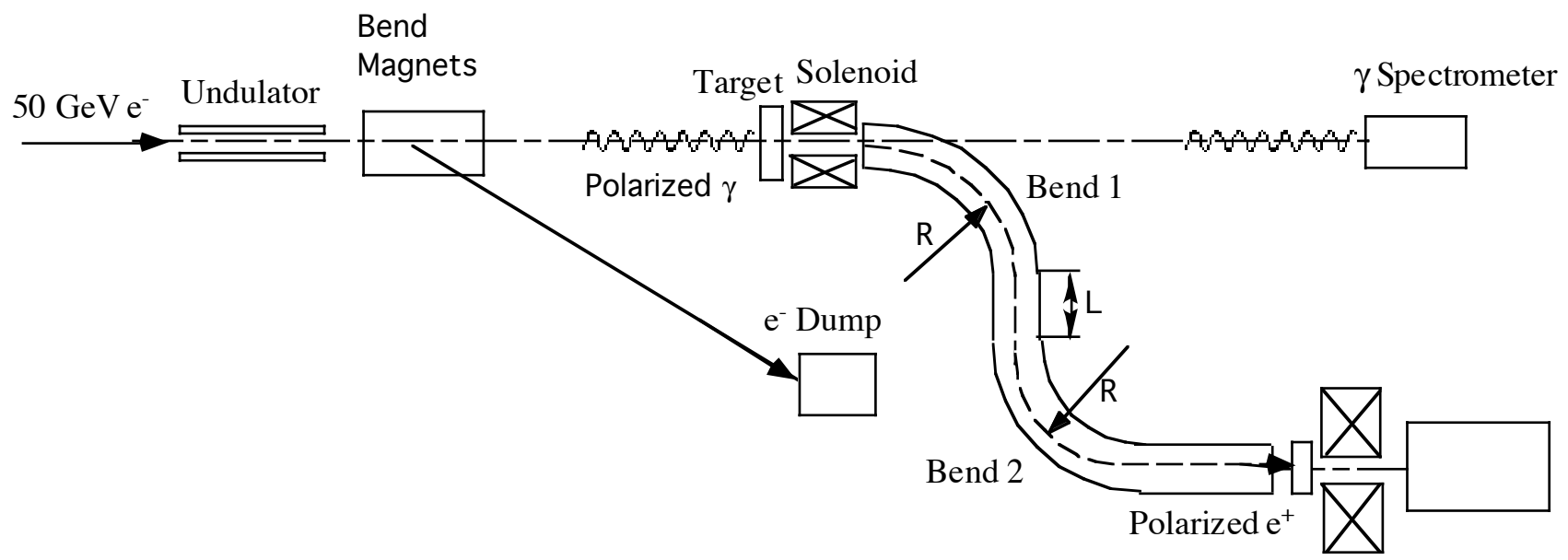

$\mathrm{e}^{+}$Spectrometer

Fig. 1. Layout of spectrometer. 

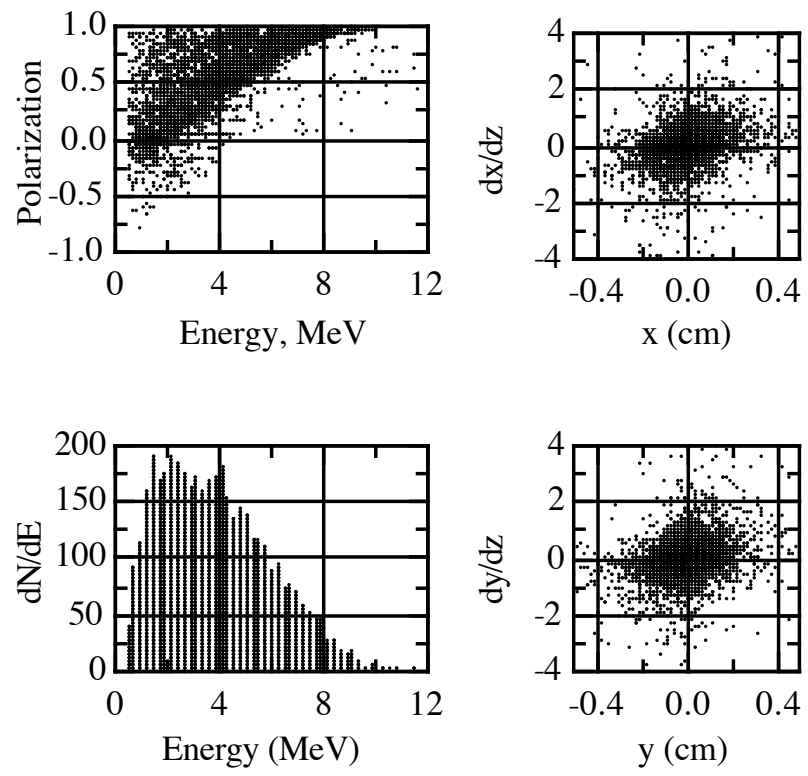

Fig. 2. Positron distribution after target. 
(a)
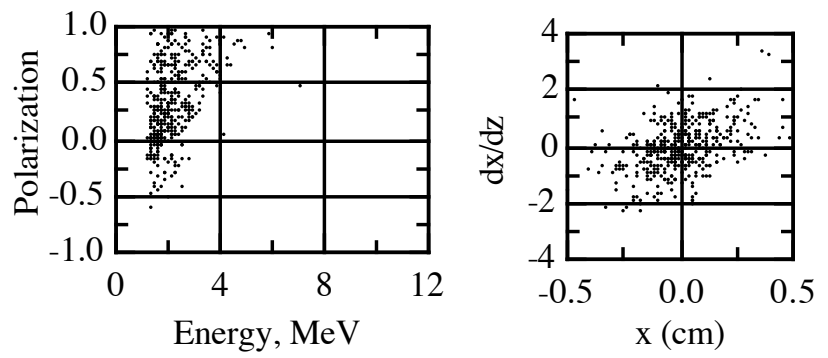

(b)
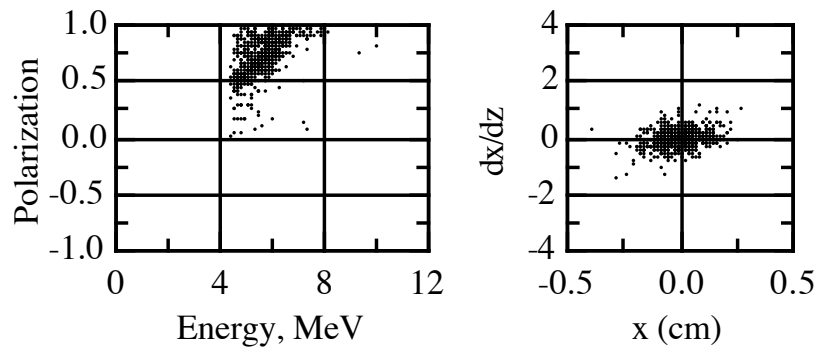

(c)
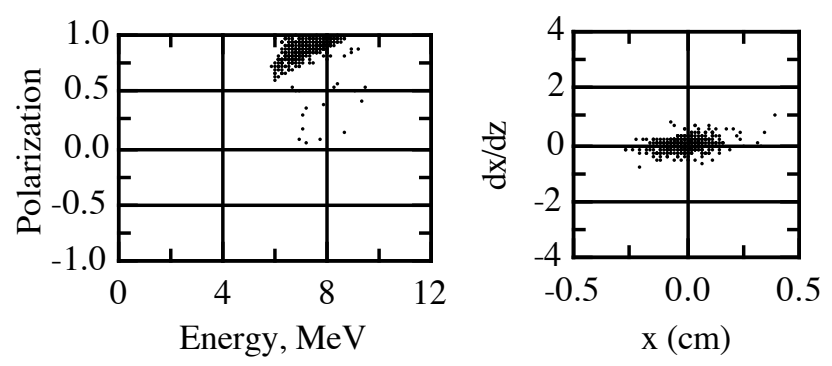

Fig. 3. Fraction of initial positron distribution with different average energy, $\overline{\mathrm{E}}$, and $\Delta \mathrm{E} / \overline{\mathrm{E}}= \pm 0.15$ :

(a) $\overline{\mathrm{E}}=1.9 \mathrm{MeV}$, (b), $\overline{\mathrm{E}}=5.3 \mathrm{MeV}$, (c) $\overline{\mathrm{E}}=7.5 \mathrm{MeV}$. 

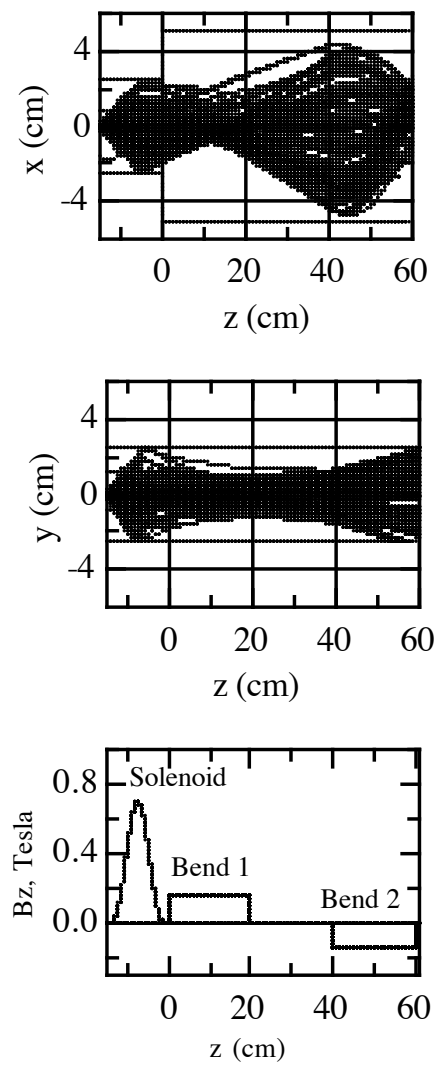

Fig. 4. Particle trajectories in the spectrometer. 


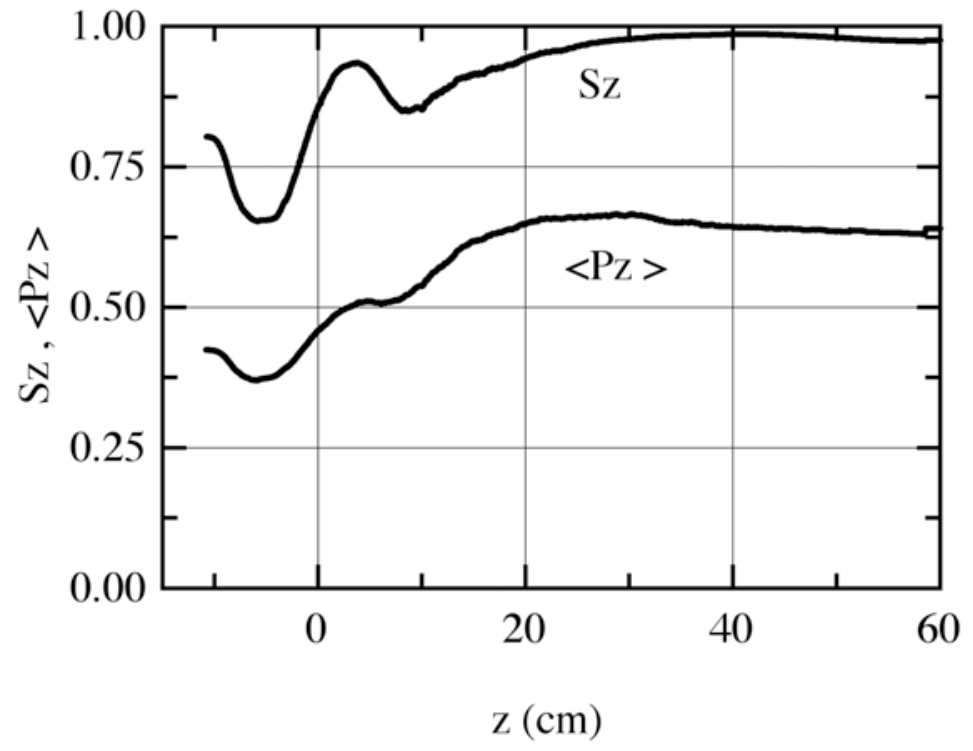

Fig. 5. Average values of longitudinal polarization, $\left\langle\mathrm{P}_{\mathrm{z}}>\right.$, and average value of $\mathrm{S}_{\mathrm{z}}$ as functions of longitudinal position. 

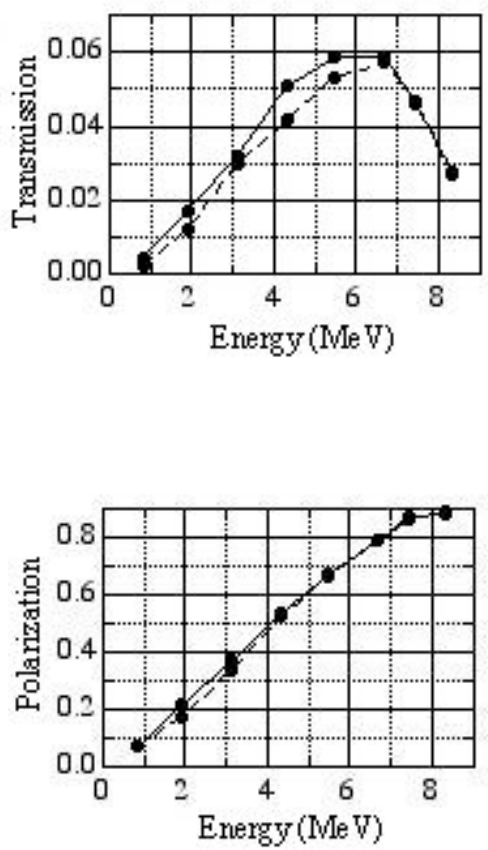

Fig. 6. Positron transmission and polarization as functions of energy of transmitted positron beam in spectrometer with field index $n=0$ : (solid) - numerical calculations, (dotted) - analytical model. 


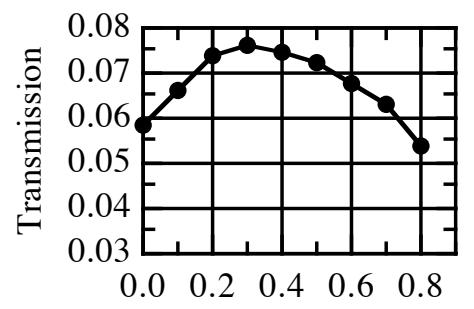

Field Index

Fig. 7. Positron transmission as a function of field index, Eq. (3.7). 